\title{
Research Paper: The Outcome of the Reconstruction Surgery to Manage the Chronic Collateral Ligament Injury of the Thumb Metacarpophalangeal Joint
}

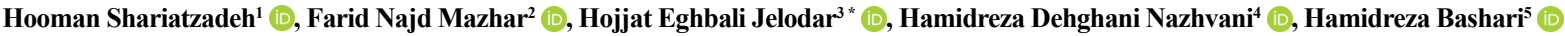

1. Bone and Joint Reconstruction Research Center, Shafa Orthopedic Hospital, Iran University of Medical Siences, Tehran, Iran

\begin{tabular}{|c|c|}
\hline $\begin{array}{l}\text { Use vour device toscan } \\
\text { and read the artice online }\end{array}$ & Iftation Shariatzadeh H, Najd Mazhar F, Eghbali Jelodar H, Dehghani Nazhvani H, Bashari H. The Outcome of the Re- \\
\hline 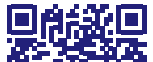 & construction Surgery to Manage the Chronic Collateral Ligament Injury of the Thumb Metacarpophalangeal Joint. Journal of \\
\hline 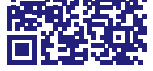 & doit: http://dx.doi.org/10.32598/JROSJ.7.1.7 \\
\hline
\end{tabular}

\section{(1) (8)}

Article info:

Received: 23 Jul 2019

Revised: 10 Aug 2019

Accepted: 12 Dec 2019

Available Online: $01 \mathrm{Feb} 2020$

Keywords:

Ulnar collateral ligament, Radial collateral ligament, Metacarpophalangeal joint, Thumb, Reconstruction surgery

\begin{abstract}
A B S T RA C T
Background: Management of chronic collateral ligament injury of the Metacarpophalangeal (MCP) joint, either its Ulnar Collateral Ligament (UCL) or Radial Collateral Ligament (RCL), is challenging.

Objectives: In this study, we report the outcome of ligament reconstruction procedure in the treatment of such injuries.

Methods: In a retrospective study, the outcomes of static reconstruction surgery of the symptomatic chronic collateral ligament injury of the thumb MCP joint (five RCL and three UCL) were evaluated. We used palmaris longus tendon in four surgeries and flexor carpi radialis tendon in the remaining. The outcome measures included the thumb range of motion, the disabilities of the arm, shoulder, and hand (QuickDASH) questionnaire, pinch and grip strength, visual analog scale, and Kapandji opposition score.

Results: Eight patients ( 6 males and 2 females) with the mean \pm SD age of $32 \pm 7.1$ years were included in this study. The Mean \pm SD duration from the injury to surgery was $66.7 \pm 99.5$ weeks. The Mean \pm SD follow-up of the patients was $4.88 \pm 1.35$ years. The mean pinch and grip strength of the involved hand averaged $98.9 \%$ and $100 \%$ of the other hand, respectively. Postoperative pain was only noticed in two patients. The Mean \pm SD postoperative Kapandji opposition score was $9.4 \pm 0.7$ (range: $8-10$ ). The Mean \pm SD QuickDASH score was $12.9 \pm 1.9$ (range: 11-17). The thumb range of motion was similar to the contralateral hand in all but one patient. All the operated joints were stable. A case of mild degenerative joint disease was the only observed postoperative complication.

Conclusion: Static reconstruction of chronic collateral ligament injury of the thumb MCP joint provides acceptable results, regardless of the time interval between the injury and surgery.
\end{abstract}

\footnotetext{
* Corresponding Author:

Hojjat Eghbali Jelodar, MD.

Address: Bone and Joint Reconstruction Research Center, Shafa Orthopedic Hospital, Iran University of Medical Siences, Tehran, Iran.

Phone: +98 (914) 4511335

E-mail: eghbalimd9@gmail.com
} 


\section{Introduction}

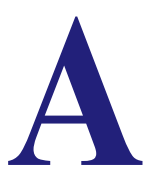

Variety of hand injuries may cause significant disability for the affected patients, particularly if initially mistreated or disregarded [1-3]. Thumbs' injuries deserve special attention as they perform fine and dexterous tasks and play a crucial role in grip function. Two main ligaments of Ulnar Collateral Ligament (UCL) and Radial Collateral Ligament (RCL) support the Metacarpophalangeal (MCP) joint of the thumb. Management of the injury of these ligaments is challenging and depends on several factors with a wide range of treatment options for each ligament. These injuries could result in symptomatic joint instability, pain, weakness, and arthritis if ignored or not adequately addressed [4].

Surgical options for symptomatic patients depend upon the time past the injury. For injuries less than three weeks past the date of injury, primary repair of the ligament is traditionally recommended. For injuries more than three weeks, reconstruction is generally advocated [5], because the formation of scar tissue and degeneration of ligament ends might undermine the direct repair of UCL and RCL $[5,6]$.

Various reconstructive surgeries have been introduced for the treatment of chronic collateral ligament injury of MCP joints of the hand. These reconstruction techniques could be classified into the dynamic and static control of joint instability. The dynamic procedure utilizes the abductor advancement and capsular reefing. While dynamic reconstruction is technically easier to perform, it provides less stability than the static method. Therefore, the static technique is more frequently employed; it involves the collateral ligament reconstruction with tendon grafting [4].

In this study, we aim to report the outcome of static reconstructive surgery of chronic collateral ligament of the MCP joint of the thumb in a series of symptomatic patients with either UCL or RCL injury.

\section{Methods}

Patients with symptomatic chronic collateral ligament injury of MCP joint of thump who were referred to our university hospital between 2012 and 2016 and underwent a triangular static ligament reconstruction surgery were included in this study. The indication for surgery included the functional deficit and pain. After the review of the electronic files, ten patients were identified as eli- gible for the study. These patients were called and asked to attend a final evaluation session. Eight patients participated in the last evaluation session and included in the final analysis.

At the final evaluation session, the function of hand was assessed with the disabilities of the arm, shoulder, and hand (QuickDASH) questionnaire in Persian [7]. The postoperative pain was measured by Visual Analog Scale (VAS). The Kapandji opposition score was used for the assessment of the thumb opposition [8]. Pinch and grip strength was assessed using a dynamometer (Hydraulic Hand Dynamometer, SH5001, SAEHAN Corporation, Korea). The thumb range of motion (flexion and extension) was also measured with a goniometer and compared with the contralateral thumb. Anteroposterior and lateral plain radiographs were also obtained to check the presence of Degenerative Joint Disease (DJD).

\section{Surgical technique}

Out of 8 reconstructive operations, 5 were performed for the reconstruction of RCL, and 3 for the reconstruction of UCL. We used palmaris longus tendon in four operations and flexor carpi radialis tendon in the remaining patients, as the palmaris longus tendon was not present in these patients. Through a curvilinear approach, we dissected the adductor or abductor aponeurosis and then assessed the ligamentous remnants and joint surface to see if the reconstruction is necessary. After deciding to perform reconstruction, a palmaris longus or a strip of flexor carpi radialis tendon was harvested. Then we triangulated the graft in the ulnar or radial side of the thumb MCP joint, as earlier described by Glickel and associates [9].

Briefly, we made a 2.8-mm drill in the base of the first phalanx (extended from palmar to dorsal) and a 3.5-mm drill on the metacarpal head (extended from ulnar to radial). After that, we treaded the tendon graft through the holes at the base of the proximal phalanx. The two graft ends were then passed together through the prepared hole of the metacarpal head (Figure 1). Intraoperatively, the tension was adjusted to ensure the full MCP joint range of motion. In the end, we repositioned the dissected muscles and closed the wound. After the surgery, we used a thumb spica cast to immobilize the thumb for 6 weeks. The cast was removed afterward, and the rehabilitation was started. 


\section{Results}

The study sample included 6 men and 2 women with the Mean \pm SD age of $32 \pm 7.1$ years (range: $23-46$ years). The Mean \pm SD duration from injury to the operation was 66.7 \pm 99.5 weeks (range: $8-300$ weeks). In 6 patients, the dominant and in 2 patients, the non-dominant hand was involved. The Mean \pm SD follow-up of the patients was $4.88 \pm 1.35$ years (range: $3-7$ years). Table 1 presents the demographic, clinical, and surgical characteristics of the patients.

The mean pinch strength of the involved hand was $98.9 \%$ of the pinch strength of the other hand. The mean grip strength of the affected hand was $100 \%$ of the grip strength of the other hand. Postoperative pain was only noticed in two patients. The Mean \pm SD Kapandji opposition score was $9.4 \pm 0.7$ (range: 8 -10). The Mean \pm SD QuickDASH score was $12.9 \pm 1.9$ (range: 11-17). The thumb range of motion was similar to the contralateral hand in all but one patient (case 8). This patient had a $30^{\circ}$ flexion contracture and limited thumb extension. The other outcome measures, including Kapandji opposition score, VAS, and QuickDASH score, were inferior in this patient, as well. Table 2 presents the detailed outcome measures of the patients.

All the operated joints were stable at the final evaluation session. Only one case of mild DJD was noticed in

Table 1. The demographic, clinical, and surgical characteristics of the patients

\begin{tabular}{|c|c|c|c|c|c|c|c|}
\hline ID & Sex & Age (y) & Involved Hand & Delay to Surgery (wk) & Affected Ligament & Graft Type & Follow-Up (y) \\
\hline 1 & Male & 30 & Dominant & 52 & $\mathrm{RCL}$ & FCR & 4 \\
\hline 2 & Male & 34 & Non-dominant & 20 & UCL & PL & 4 \\
\hline 3 & Male & 32 & Dominant & 104 & UCL & PL & 4 \\
\hline 4 & Female & 23 & Dominant & 300 & $\mathrm{RCL}$ & FCR & 7 \\
\hline 5 & Female & 46 & Dominant & 28 & UCL & FCR & 3 \\
\hline 6 & Male & 33 & Non-dominant & 10 & $\mathrm{RCL}$ & PL & 6 \\
\hline 7 & Male & 24 & Dominant & 8 & $\mathrm{RCL}$ & PL & 5 \\
\hline 8 & Male & 34 & Dominant & 12 & $\mathrm{RCL}$ & FCR & 6 \\
\hline
\end{tabular}

RCL: Radial Collateral Ligament; UCL: Ulnar Collateral Ligament; FCR: Flexor Carpi Radialis; PL: Palmaris Longus

Table 2. Outcome measures of the patients

\begin{tabular}{|c|c|c|c|c|c|c|c|}
\hline ID & $\begin{array}{l}\text { Pinch Strength, } \\
\text { Involved Hand } \\
\text { (Ib) }\end{array}$ & $\begin{array}{l}\text { Pinch Strength, Con- } \\
\text { tralateral Hand (Ib) }\end{array}$ & $\begin{array}{l}\text { Grip Strength, In- } \\
\text { volved Hand (Ib) }\end{array}$ & $\begin{array}{l}\text { Grip Strength, Contra- } \\
\text { lateral Hand (Ib) }\end{array}$ & $\begin{array}{l}\text { VAS for } \\
\text { Pain }\end{array}$ & Quick DASH & $\begin{array}{c}\text { Kapandji } \\
\text { Opposition } \\
\text { Score }\end{array}$ \\
\hline 1 & 23 & 23 & 117 & 116 & 0 & 12 & 10 \\
\hline 2 & 27 & 28 & 127 & 129 & 0 & 13 & 9 \\
\hline 3 & 26 & 24 & 118 & 115 & 2 & 13 & 10 \\
\hline 4 & 16 & 15 & 72 & 70 & 0 & 12 & 10 \\
\hline 5 & 15 & 14 & 70 & 65 & 0 & 11 & 9 \\
\hline 6 & 25 & 27 & 115 & 115 & 0 & 11 & 10 \\
\hline 7 & 28 & 27 & 120 & 119 & 0 & 14 & 9 \\
\hline 8 & 16 & 20 & 100 & 110 & 6 & 17 & 8 \\
\hline
\end{tabular}

VAS: Visual Analog Scale; DASH: Disabilities of the Arm, Shoulder, and Hand. 


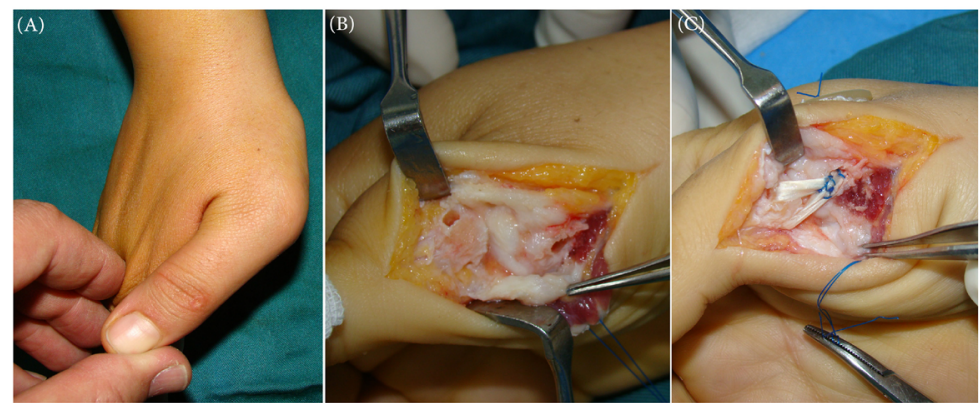

Orthopedic Science

Figure 1. A. Preoperative photography of a patient with chronic RCL injury of the thumb metacarpophalangeal joint; B. Intraoperative photography of the same patients showing the tunnel preparation step for the reconstruction purpose; C. Postreconstruction photography of the same patient

our series (case 5) (Figure 2). No other complication was recorded during the follow-up period of the study.

\section{Discussion}

In this study, we evaluated the outcome of a triangular static reconstruction technique for the management of chronic UCL/RCL injury of the thumb of MCP joints. Based on the study results, the static reconstruction technique provides a pinch and grip strength, almost similar to the unaffected hand. The postoperative function of hand and thumb opposition was also favorable following this technique. Moreover, the thumb range of motion could be recovered in the majority of patients.

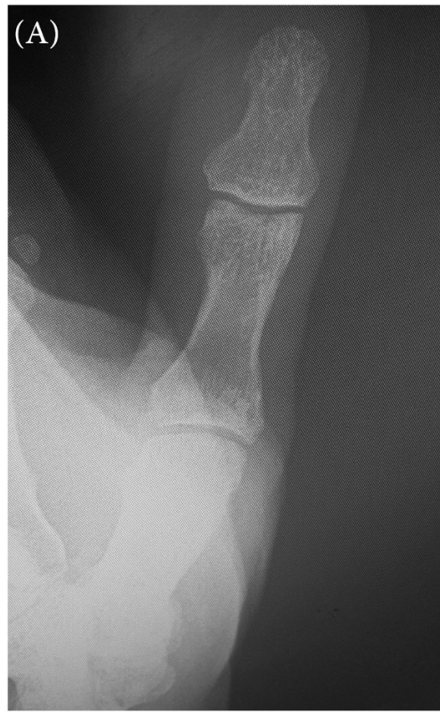

In 1993, Glickel et al. presented the ligament replacement technique for the management of chronic UCL instability of thumb MCP joint in 26 patients. At a mean follow-up of 4.5 years, the key pinch strength of the involved hand averaged $95.2 \%$ of the non-involved hand. Arc of motion was retained in $85 \%$ of patients. The results were reported as excellent in 20 patients, good in four patients, and fair in two patients. Accordingly, they introduced this technique as a successful approach in restoring stability, reducing pain, with minimal loss of motion [9]. Consequently, the methods were implicated in later workouts.

Catalano et al. assessed the efficacy of late reconstruction for the management of 10 chronic RCL injuries of thumb MCP joint. The surgery was performed at a

Figure 2. A. Anteroposterior radiograph of the thumb metacarpophalangeal joint seven years after RCL reconstruction showing no degenerative changes; $\mathrm{B}$. Anteroposterior radiograph of the thumb metacarpophalangeal joint three years after UCL reconstruction showing a mild degenerative joint disease 
mean duration of 6.8 months after the injury. The mean follow-up of the patients was 6 months. Based on their report, excellent and good results were observed in 8 and 2 patients, respectively. The overall satisfaction was excellent in all patients. They concluded that the reconstruction of chronic RCL injuries of thumb MCP joint provides similar results to repair of the acute RCL injuries of thumb MCP joint [10]. The mean delay to surgery was 16.8 months in the present series, which was considerably more than that of Catalano et al. study. Even though the results were comparable to the study of Catalano et al., 7 out of 8 patients of the present series had an excellent outcome.

Martínez-Villén et al. reported the results of static triangular ligament reconstruction in thumb MCP joint chronic posttraumatic instability (7 UCL and 3 RCL reconstructions). The mean delay from injury to surgery was 78.9 months. With a mean follow-up of 40.2 months, the mean thumb MCP joint stress testing of the involved hand was only $6.5^{\circ} \mathrm{C}$ less than the non-involved hand. The mean range of flexion and extension were respectively $10.5^{\circ}$ and $8^{\circ}$ lower than the contralateral hand. The Kapandji opposition score, grip, and pinch strength were minimally different from the healthy hand. All patients returned to their daily activities [11].

The delay to surgery was remarkably more in the study of Martínez-Villén and associates. However, similar to their study, the Kapandji opposition score, grip and pinch strength of the involved hand were minimally different from the healthy hand in the present series. These results reveal that the reconstruction of collateral ligament of thumb MCP joint will result in a favorable outcome, no matter how long has passed from the date of injury.

Oka et al. also reported the results of the reconstruction of chronic injuries to the collateral ligament of MCP joints in 13 patients (thumb/UCL, 10 cases; thumb/RCL, 2 cases; and middle finger/RCL, 1 case). All patients had pain before the surgery. The mean preoperative instability was $41^{\circ}$. With an average follow-up of 5.5 years, the pain and joint instability were completely resolved in all patients. However, the mean $\mathrm{MCP}$ joint range of motion was $1^{\circ} \mathrm{C}$ on extension and $58^{\circ} \mathrm{C}$ on flexion [12]. Loss of flexion and extension following the reconstruction of collateral ligaments of the thumb MCP joint has also been frequently reported by other authors [13-17]. Contrary to the most previous investigations, the present study revealed that the reconstruction of chronic injuries to the collateral ligaments of the thumb MCP joint could provide a symmetric range of motion to the contralateral hand in the majority of patients.
Over-correction of instability could be regarded as one of the potential consequences of this surgery that may be caused by the over-tensioning of the ligamentoplasty. Fusetti et al. reported $9^{\circ}$ excess of laxity on postoperative radial stress testing compared with the non-involved thumb [18]. All the operated joints of the present study were stable in the last evaluation session.

The results of the present study and those of the earlier investigations reveal that chronic reconstruction of collateral ligaments of the thumb MCP joint provides acceptable results, regardless of the delay period from the injury to surgery. The present study has some limitations. The main limitation of this study was the small sample size that weakens the statistical analysis of the results. Further studies with a larger sample size are required to confirm the study results.

\section{Conclusion}

Static reconstruction of the chronic collateral ligament injuries of the thumb MCP joint could provide an acceptable functional outcome, pinch, and grip strength, and thumb opposition, regardless of the time interval from the injury to the surgery. Although the postoperative restricted thumb range of motion and instability was reported in several earlier investigations, we observed stable joints with a symmetric range of motion to the contralateral hand in the majority of the patients. Therefore, we recommend this reconstruction approach for the management of similar injuries in future workouts.

\section{Ethical Considerations}

\section{Compliance with ethical guidelines}

The Review Board of our institute approved this retrospective study (Code: IR.BJRC.ER.1397.386), and written consent was obtained from the patients to use their medical data for publication.

\section{Funding}

This research did not receive any specific grant from funding agencies in the public, commercial, or not-forprofit sectors.

\section{Authors' contributions}

All authors contributed in preparing this article.

\section{Conflict of interest}

The authors declared no conflict of interest. 


\section{References}

[1] Najd Mazhar F, Jafari D, Taraz H, Mirzaei A. Treatment of dorsal fracture-dislocations of the proximal interphalangeal joint using the shotgun approach. J Hand Surg Eur Vol. 2018; 43(5):499-505. [DOI:10.1177/1753193418766274] [PMID]

[2] Najd Mazhar F, Ebrahimi H, Jafari D, Mirzaei A. Radial head resection versus prosthetic arthroplasty in terrible triad injury: A retrospective comparative cohort study. Bone Joint J. 2018; 100(11):1499-505. [DOI:10.1302/0301620X.100B11.BJJ-2018-0293.R1] [PMID]

[3] Najd Mazhar F. Proximal interphalangeal joint fracture dislocation. Shafa Orthopedic Journal. 2014; 1(2):29-33 .

[4] Patel S, Potty A, Taylor EJ, Sorene ED. Collateral ligament injuries of the metacarpophalangeal joint of the thumb: a treatment algorithm. Strategies Trauma Limb Reconstr. 2010; 5(1):1-10. [DOI:10.1007/s11751-010-0079-7] [PMID] [PMCID]

[5] Smith RJ. Posttraumatic instability of the metacarpophalangeal joint of the thumb. J Bone Joint Surg Am. 1977; 59(1):14-21. [DOI:10.2106/00004623-19775901000002] [PMID]

[6] Kaplan EB. The pathology and treatment of radial subluxation of the thumb with ulnar displacement of the head of the first metacarpal. JBJS. 1961; 43(4):541-6. [DOI:10.2106/00004623-196143040-00010]

[7] Mousavi SJ, Parnianpour M, Abedi M, Askary-Ashtiani A, Karimi A, Khorsandi A, et al. Cultural adaptation and validation of the Persian version of the Disabilities of the Arm, Shoulder and Hand (DASH) outcome measure. Clin rehab. 2008; 22(8):749-57. [DOI:10.1177/0269215508085821] [PMID]

[8] Kapandji I. Biomechanics of the thumb. Hand. 1981; 1:40422.

[9] Glickel SZ, Malerich M, Pearce SM, Littler JW. Ligament replacement for chronic instability of the ulnar collateral ligament of the metacarpophalangeal joint of the thumb. J Hand Surg Am. 1993; 18(5):930-41. [DOI:10.1016/03635023(93)90068-E] [PMID]

[10] Catalano III LW, Cardon L, Patenaude N, Barron OA, Glickel SZ. Results of surgical treatment of acute and chronic grade III [corrected] tears of the radial collateral ligament of the thumb metacarpophalangeal joint. J Hand Surg Am. 2006; 31(1):68-75. [DOI:10.1016/j. jhsa.2005.08.013] [PMID]

[11] Martínez-Villén G, García JP, Barrero PP, Herrera A. Thumb metacarpophalangeal joint ligament reconstruction with a triangular tendon graft in posttraumatic chronic instability. Chir Main. 2012; 31(1):1-6. [DOI:10.1016/j. main.2012.01.006] [PMID]

[12] Oka Y, Harayama H, Ikeda M. Reconstructive procedure to repair chronic injuries to the collateral ligament of metacarpophalangeal joints of the hand. Hand Surg. 2003; 8(1):81-5. [DOI:10.1142/S021881040300156X] [PMID]

[13] Durham JW, Khuri S, Kim MH. Acute and late radial collateral ligament injuries of the thumb metacarpophalangeal joint. J Hand Surg Am. 1993; 18(2):232-7. [DOI:10.1016/0363-5023(93)90353-5] [PMID]
[14] Camp RA, Weatherwax RJ, Miller EB. Chronic posttraumatic radial instability of the thumb metacarpophalangeal joint. J Hand Surg Am. 1980; 5(3):221-5. [DOI:10.1016/ S0363-5023(80)80006-2]

[15] Breek J, Tan A, Van Thiel TP, Daantje C. Free tendon grafting to repair the metacarpophalangeal joint of the thumb. Surgical techniques and a review of 70 patients. J Bone Joint Surg Br. 1989; 71(3):383-7. [DOI:10.1302/0301620X.71B3.2722925] [PMID]

[16] Mitsionis GI, Varitimidis SE, Sotereanos GG. Treatment of chronic injuries of the ulnar collateral ligament of the thumb using a free tendon graft and bone suture anchors. J Hand Surg Br. 2000; 25(2):208-11. [DOI:10.1054/ jhsb.1999.0353] [PMID]

[17] Lee SK, Kubiak EN, Lawler E, Iesaka K, Liporace FA, Green SM. Thumb metacarpophalangeal ulnar collateral ligament injuries: A biomechanical simulation study of four static reconstructions. J Hand Surg Am. 2005; 30(5):1056-60. [DOI:10.1016/j.jhsa.2005.05.004] [PMID]

[18] Fusetti C, Papaloizos M, Meyer H, Borisch N, Della Santa D. The ECRL bone-tendon ligamentoplasty for chronic ulnar instability of the metacarpophalangeal joint of the thumb. Chir Main. 2005; 24(5):217-21. [DOI:10.1016/j. main.2005.08.003] [PMID] 